\title{
Rhabdomyolysis after Cytoreductive Surgery and Hyperthermic Intraperitoneal Chemotherapy: A Case Report
}

\author{
Rob Bielen $^{a} \quad$ Geert Verswijvel $^{b} \quad$ Kurt Van der Speeten ${ }^{a, c}$ \\ Departments of a Surgical Oncology and ${ }^{b}$ Radiology, Ziekenhuis Oost-Limburg, Genk, and \\ 'Biomed Research Institute, Faculty of Medicine, University Hasselt, Diepenbeek, Belgium
}

\section{Key Words}

Gastric cancer · Peritoneal carcinomatosis · Cytoreductive surgery · Hyperthermic intraperitoneal perioperative chemotherapy · Rhabdomyolysis · Compartment syndrome

\begin{abstract}
Gastric cancer with peritoneal carcinomatosis is a disease with a poor prognosis. Cytoreductive surgery (CRS) and hyperthermic intraperitoneal perioperative chemotherapy (HIPEC) can improve prognosis, although in most cases this should still be considered as a palliative treatment. Therefore, morbidity has to be avoided at all cost as quality of life is of utmost importance. We describe the case of a 64-year-old female with an adenocarcinoma of the stomach that was initially treated with a Billroth II gastrectomy, adjuvant chemotherapy and radiotherapy. During follow-up, the diagnosis of peritoneal carcinomatosis was made, and the patient was referred for CRS and HIPEC. Postoperatively, she developed rhabdomyolysis in both gastrocnemius muscles. Renal function remained within normal limits, but ultrasonography of the lower legs suggested the presence of bilateral abscesses. Drainage with pigtail catheters was necessary for more than 1 month, significantly impairing quality of life. The objective of this case report is to heighten awareness for this complication. Rhabdomyolysis is a rare complication of CRS and HIPEC, with a significant impact on quality of life. Prevention is necessary and can be achieved by adequate surgical positioning, using the altered lithotomy position, sufficient padding and by preventing hypovolemia.
\end{abstract}


Bielen et al.: Rhabdomyolysis after Cytoreductive Surgery and Hyperthermic Intraperitoneal Chemotherapy: A Case Report

\section{Background}

Gastric cancer with peritoneal carcinomatosis (PC) has a poor prognosis. Median survival with systemic treatment is reported to be less than 3 months [1]. In the past, cytoreductive surgery (CRS) combined with hyperthermic intraperitoneal perioperative chemotherapy (HIPEC) improved prognosis of patients with isolated PC from other primary tumors [2-5]. In locally advanced gastric cancer with serosal or lymph node invasion, but without established PC, CRS + HIPEC was also associated with a better outcome according to three randomized controlled trials [6-8]. The same concept was then applied to gastric cancer with established PC. Several studies suggested that CRS and HIPEC could improve survival of these patients, with an acceptable morbidity and mortality $[9,10]$. Patient selection is of utmost importance [11,12]. Preoperative imaging and intraoperative staging systems estimating the extent of PC correlate with morbidity and mortality [13, 14]. Completeness of cytoreduction (CCR) and the experience of the surgical team also influence the outcome significantly, improving survival and lowering the risk of complications [15].

Complications relate to both surgery and chemotherapy [16]. We report on a rare but important complication namely rhabdomyolysis. The reported incidence of rhabdomyolysis after surgery varies widely between $0.67 \%$ in laparoscopic nephrectomy and $37-77 \%$ in gastric bypass $[17,18]$. The use of different diagnostic criteria is responsible for the wide variation between these data. In the first article, rhabdomyolysis was only diagnosed after patients' complaints, whereas in the second article, creatinine kinase (CK) values were measured in all patients and rhabdomyolysis was defined as an increase of more than 5 times the upper limit. Nevertheless, body mass index is recognized as a significant risk factor [18]. Prolonged surgery and certain surgical positions are also identified risk factors for rhabdomyolysis [19]. The aim of this report is to heighten awareness for this complication.

\section{Case Description}

\section{Presentation}

A 64-year-old female presented with an invasive adenocarcinoma of the stomach. She was treated with a Billroth II gastrectomy, followed by radiotherapy and chemotherapy. Pathology reported invasion of both lymphatic and blood vessels (T3N2M0). Adjuvant treatment was well tolerated. Six months later, the patient started complaining about diffuse abdominal pain. Initially, no focus was identified. Biochemistry remained within normal limits. Another 4 months later, a follow-up CT suggested peritoneal recurrence of the gastric carcinoma. PC was described caudal from the right hepatic lobe and anterior of the distal one-third of the sigmoid. Enlarged lymph nodes were demonstrated para-aortic. Chest X-ray was normal. A PET-CT was not executed because of the low sensitivity and specificity in gastric cancer, especially after gastrectomy [20].

\section{Treatment}

The patient was referred for CRS and HIPEC after multidisciplinary consultation. She was placed in the modified lithotomy position and had intermittent compression stockings on both legs to improve venous return. Induction of anesthesia included the administration of propofol, fentanyl and rocuronium bromide intravenously. Anesthesia was maintained with target-controlled infusion of propofol. The peritoneal carcinomatosis index (PCI) according to Sugarbaker added up to a total score of 12 [21, 22]. A complete cytoreduction (CCR 0.1) was achieved. Then, HIPEC was administered by the open technique. The 
peritoneum was perfused with icodextrin $1.5 \%$ until a uniform intraperitoneal temperature of $41.5^{\circ} \mathrm{C}$ was managed. Subsequently, $78 \mathrm{mg}$ of cisplatinum $\left(50 \mathrm{mg} / \mathrm{m}^{2}\right)$ and $23.4 \mathrm{mg}$ of doxorubicin $\left(15 \mathrm{mg} / \mathrm{m}^{2}\right)$ were administered in three doses over a total of $90 \mathrm{~min}$. During the operation, the patient stayed hemodynamically stable with a total blood loss of $900 \mathrm{ml}$. Fluid loss was compensated by $4,500 \mathrm{ml}$ of crystalloid, $2,000 \mathrm{ml}$ of colloid and $350 \mathrm{ml}$ of whole blood transfusion. Diuresis remained above $1 \mathrm{ml} / \mathrm{kg} / \mathrm{h}$ all the time. The total operating time was $10 \mathrm{~h}$ and the anesthetic time was $10 \mathrm{~h}$ and $50 \mathrm{~min}$. The patient was transferred to the intensive care unit in a stable condition.

\section{Postoperative Course}

During the first $24 \mathrm{~h}$ postoperatively, the patient had a urine output of 1,500 ml. Biochemistry revealed an elevated serum CK of 2,175 U/l (normal range: 26-140 U/l) on day 0, which is not unusual after CRS and HIPEC. However, on postoperative day 1, CK levels further increased to a maximum of 9,079 $\mathrm{U} / \mathrm{l}$ and urine testing revealed a myoglobinuria. The diagnosis of rhabdomyolysis was made. After removal of the peridural catheter on day 2 , the patient started to complain of severe pain in both calves and paresthesias in her feet. Clinical examination of the lower legs demonstrated normal plantar and dorsiflexion, and normal arterial pulsations, excluding a compartment syndrome. Venous duplex ultrasonography excluded deep venous thrombosis but demonstrated hyperechoic areas within the muscles (fig. 1). Monitoring of the CK levels showed a gradual decline to $190 \mathrm{U} / \mathrm{l}$ on day 9. Renal function stayed within normal limits the whole time. Later on, the patient developed bilateral sterile abscesses in the gastrocnemius muscles as a complication of the rhabdomyolysis. The abscesses were punctured and drained with a pigtail catheter under the guidance of ultrasonography (fig. 2, fig. 3). Eventually the drains were removed, and the patient could be discharged after 1 month and 10 days. She was readmitted after 3 days with the same complaints, and a new ultrasonography-guided puncture was performed. Analgesics were administered if necessary, and after 8 more days in the hospital, the patient went home with the drains in situ. After 2 weeks, the catheters were permanently removed at the outpatient clinic.

\section{Discussion}

Gastric cancer with PC is a disease with a poor prognosis [1]. CRS and HIPEC can improve prognosis with acceptable mortality, morbidity and quality of life $[9,10,23,24]$. In 2009, Glehen et al. [3] reported a multicenter cohort study of 1,290 patients with PC. CRS combined with HIPEC could achieve long-term survival in selected patients. However, they reported differences in outcome according to the primary tumor that caused PC. Gastric cancer had the poorest prognosis. In a subanalysis, Glehen et al. [9] investigated specifically those patients with PC from gastric cancer. They demonstrated a median survival of 9.2 months, which increased to 15 months in patients with CCR 0.1. In a phase III randomized clinical trial of patients with PC from gastric cancer, Yang et al. [10] reported medium survivals of 11.0 months in the CRS and HIPEC group, opposite to 6.5 months for CRS alone. In 2011, Gill et al. [24] reviewed all previous studies of CRS and HIPEC as a treatment for PC from gastric cancer. A total of ten studies were included, and analysis demonstrated a median survival of 7.9 months. Survival increased to 15 months for patients with CCR 0.1. Thus, it is possible to obtain a significant increase of survival. Patient selection is essential as CCR after CRS and HIPEC is the most important prognostic factor regardless of the origin of PC [11-13]. 
However, at present, CRS combined with HIPEC should be considered as a palliative treatment in most patients with PC from gastric cancer. Next to survival, morbidity and quality of life become equally important in this setting. Morbidity is graded according to the National Common Terminology Criteria for Adverse Events (NCTCAE ${ }^{\circledR}$ ) [25]. In a review of morbidity after CRS and HIPEC, Glockzin et al. [16] state that the incidence of major morbidity (grades III and IV) is relatively high, but comparable to the incidence of morbidity in any other major surgery. Several independent factors, such as CCR, PCI and experience of the surgical team, are correlated with morbidity and mortality. CCR 0.1 not only improves survival, but is also associated with a lower morbidity [9-11]. Low PCI scores increase the feasibility of CCR 0.1 , with possibly less extensive surgery $[14,26]$. The experience of the surgical team influences the risk of morbidity, as there is a long learning curve for CRS and HIPEC [27]. Postoperative morbidity lowers quality of life. When patients recover from CRS + HIPEC, quality of life will also restore, and after 3-4 months, most patients return to baseline or better functioning $[28,29]$. However, postoperative complications can significantly decrease quality of life, and this must be avoided at all times.

Most complications are surgery-related and/or a consequence of chemotherapy. In this case, we present a rare complication namely rhabdomyolysis of the lower legs. Rhabdomyolysis is defined as necrosis of skeletal muscle fibers with release of the fiber contents into the blood and urine [30]. There are many different etiologies for this condition: traumatic crush injury, hereditary muscle enzyme defects, several drugs and toxins, as well as metabolic endocrine disorders [31]. Another important etiology is prolonged immobilization, as in surgery. Persistent muscle hypoxemia due to unrelieved pressure on gravitydependent body parts leads to a depletion of adenosine triphosphate (ATP) in the myocytes. Malfunction of a series of pumps and channels that regulate intracellular calcium levels, leads to an unregulated increase in intracellular calcium. This results in the activation of calcium-dependent neutral proteases, followed by destruction of myofibrillar, cytoskeletal and membrane proteins, ending in the disintegration of the myocytes [32]. The necrosis of muscle cells and loss of capillary wall integrity then results in transudation and exudation, ending in massive edema within the muscle compartment [33]. The edema increases the pressure within the compartment, affecting the vascular supply and forming a vicious cycle $[21,34,35]$. Further, when surgical procedures are terminated, ischemia-reperfusion injury and the following localized tissue edema can result in a continued low perfusion state in the local tissue bed [30,33]. Additionally, a systemic hypovolemia is produced through the loss of intravascular fluid in local tissue beds further compromising perfusion. Finally, because of reperfusion, large quantities of myoglobin, potassium, phosphate, CK, lactate dehydrogenase and urate leak into the circulation, potentially leading to acute renal failure, cardiotoxicity or other complications [19, 33, 35]. This condition must not be confused with compartment syndrome. In compartment syndrome, high pressures within a muscle compartment compromise vascular supply [36, 37]. This leads to loss of peripheral arterial pulsations and neurological symptoms like paresthesias, muscular weakness or paralysis. A compartment syndrome can lead to rhabdomyolysis, and vice versa [19, 38]. However, compartment syndrome and rhabdomyolysis also exist separately. Further investigation is necessary to identify the factors that determine the exact relationship between rhabdomyolysis and compartment syndrome.

Rhabdomyolysis has been described as a complication of renal, neurosurgical and bariatric surgery [17, 18, 38-42]. Several risk factors are reported: obesity, prolonged duration of surgery, surgical positioning, hypovolemia, diabetes and hypertension. Firstly, morbid obesity is a major risk factor, as a body mass index of more than $30 \%$ of the ideal already increases the risk of developing rhabdomyolysis [41]. The extra weight puts substantial 
pressure on the muscle mass [42]. Other mechanisms related to the metabolic derangement could also be involved $[18,42]$. Secondly, prolonged duration of surgery is correlated with a higher incidence of rhabdomyolysis. In 1986, a study of Harris et al. [43] demonstrated that skeletal muscle tolerates ischemia for $2 \mathrm{~h}$. Thereafter, ultrastructural ischemic changes occur. After 4-6 h, these changes become irreversible [44]. Thirdly, surgical positioning is also important. Various case reports in multiple disciplines describe rhabdomyolysis after prolonged surgery in different positions [17-19, 38, 41, 45]. They all indicate a correlation between surgical position, surgical length and the risk of rhabdomyolysis. Accordingly, prolonged pressure on particular muscle groups leads to rhabdomyolysis. Finally, diabetes and hypertension may increase the risk of rhabdomyolysis by leading to chronic microcirculation abnormalities $[18,38]$. This leads to a higher susceptibility to perfusion problems and is a predisposing factor for rhabdomyolysis.

The outcome of rhabdomyolysis is correlated to an early diagnosis [46, 47]. A high level of awareness is crucial. Treatment is focused on preserving renal function. Because of the tendency to develop hypovolemia, aggressive fluid replacement is essential [33, 39]. Urine output should be monitored by placing a urinary catheter. Then, intravenous fluids are administered by a rate of $500-1,000 \mathrm{ml} / \mathrm{h}$ to ensure a urine output greater then $150-300$ $\mathrm{ml} / \mathrm{h}[47,48]$. However, if oliguria persists, fluid overload should also be avoided. The exact composition of the fluid regimen is still under discussion. In animal studies, urinary alkalinization by using sodium bicarbonate has positive effects [49]. Empirically, the administration of mannitol is also defendable as it induces osmotic diuresis as well as renal vasodilatation and is involved in free radical scavenging, although there is a risk of acidifying the urine [50]. However, clinical superiority has not been shown for these therapies when compared to the administration of normal saline solution [51]. Another point of importance is the prevention or treatment of electrolyte disorders. Particularly the control of hyperkalemia is important to prevent cardiotoxicity. Potassium can be removed from the body by kaliuresis (loop diuretics) and intestinal potassium binders [33]. In rare cases, when the kidneys no longer respond to these supportive measures, temporary dialysis can be necessary. If a compartment syndrome is present, a decompressive fasciotomy is necessary [48].

As discussed above, CRS and HIPEC is in most cases a palliative treatment for PC from gastric cancer. Therefore, prevention of complications is important. Not only because they decrease the prognosis, but also because they lower the quality of life in patients with a shortened life expectancy. There are several hypothetical methods to prevent rhabdomyolysis in this specific setting. However, morbid obesity, diabetes and hypertension cannot be changed rapidly. Hypovolemia and surgical positioning are the only two modifiable risk factors. Hypovolemia can simply be prevented by monitoring fluid loss during the operation and replacing this loss sufficiently. To reduce the risk of rhabdomyolysis by surgical positioning, various methods are proposed. Firstly, generous padding of all pressure points effectively lowers the risk of rhabdomyolysis, if properly executed $[40,45,52]$. Secondly, the modified lithotomy position increases the risk of rhabdomyolysis and compartment syndrome of the lower legs. Therefore, this position should not be used if possible. Nevertheless, if necessary, an altered lithotomy position is proposed, with support at the feet instead of the knees or lower legs by the use of St. Mark's leg holders, thereby effectively lowering the compartment pressure [37]. Another benefit of this altered position is the impossibility of compressing the lower popliteal veins. Finally, although intermittent pneumatic stockings lower pressure in the muscle compartment, in prolonged surgery one should consider not to use them during the whole operating time [53], as they eventually become a source of friction and pressure themselves. 
Bielen et al.: Rhabdomyolysis after Cytoreductive Surgery and Hyperthermic Intraperitoneal Chemotherapy: A Case Report

\section{Conclusion}

This report presents a case of rhabdomyolysis after treatment for PC from gastric cancer with CRS and HIPEC. In most cases, this is a palliative treatment, thus it is essential that quality of life is secured. Therefore, all morbidity should be prevented. Rhabdomyolysis is a rare complication of CRS and HIPEC, but it can significantly decrease quality of life. Prevention is necessary and can be achieved by adequate surgical positioning, using the altered lithotomy position, sufficient padding and by preventing hypovolemia.

\section{Disclosure Statement}

The authors declare that they have no competing interests.

\section{References}

1 Sadeghi B, Arvieux C, Glehen 0, et al: Peritoneal carcinomatosis from non-gynecologic malignancies: results of the EVOCAPE I multicentric prospective study. Cancer 2000;88:358-363.

-2 Verwaal VJ, van Ruth S, de Bree E, van Slooten GW, van Tinteren H, Boot H, Zoetmulder F: Randomized trial of cytoreduction and hyperthermic intraperitoneal chemotherapy versus systemic chemotherapy and palliative surgery in patients with peritoneal carcinomatosis of colorectal cancer. J Clin Oncol 2003;21:3737-3743.

-3 Glehen O, Gilly FN, Boutitie F, et al: Toward curative treatment of peritoneal carcinomatosis from nonovarian origin by cytoreductive surgery combined with perioperative intraperitoneal chemotherapy. Cancer 2010;116:5608-5618.

-4 Portilla G, Barrios P, Rufian S, et al: Management of peritoneal surface malignancy with cytoreductive surgery and perioperative intraperitoneal chemotherapy. Eur J Surg Oncol 2006;32:628-631.

-5 Kusamara S, Younan R, Baratti D, Costanzo P, Favaro M, Gavazzi C, Deraco M: Cytoreductive surgery followed by intraperitoneal hyperthermic perfusion: analysis of morbidity and mortality in 209 peritoneal surface malignancies treated with closed abdomen technique. Cancer 2006;106:1144-1153.

-6 Yonemura Y, de Aretxabala X, Fujimura T, et al: Intraoperative chemothermic peritoneal perfusion as an adjuvant to gastric cancer: final results of a randomized controlled study. Hepatogastroenterology 2001;48:1176-1182.

7 Yu W, Whang I, Chung HY, Averbach A, Sugarbaker PH: Indications for early postoperative intraperitoneal chemotherapy of advanced gastric cancer: results of a prospective randomized trial. World J Surg 2001;25:985-990.

-8 Fujimoto S, Takahashi M, Mutou T, Kobayashi K, Toyosawa T: Successful intraperitoneal hyperthermic chemoperfusion for the prevention of postoperative peritoneal recurrence in patients with advanced gastric carcinoma. Cancer 1999;85:529-534.

-9 Glehen 0, Gilly FN, Arvieux C, et al: Peritoneal carcinomatosis from gastric cancer: a multi-institutional study of 159 patients treated by cytoreductive surgery combined with perioperative intraperitoneal chemotherapy. Ann Surg Oncol 2010;17:2370-2377.

-10 Yang XJ, Huang CQ, Suo T, et al: Cytoreductive surgery and hyperthermic intraperitoneal chemotherapy improves survival of patients with peritoneal carcinomatosis from gastric cancer: final results of a phase III randomized clinical trial. Ann Surg Oncol 2011;18:1575-1581.

11 Piso P, Glockzin G, von Breitenbuch P, et al: Patient selection for a curative approach to carcinomatosis. Cancer 2009;15:236-242.

12 Saxena A, Yan TD, Morris DL: Critical assessment of preoperative and operative risk factors for complications after iterative peritonectomy procedures. Eur J Surg Oncol 2010;36:309-314.

13 Cotte E, Passot G, Gilly FN, Glehen O: Selection of patients and staging of peritoneal surface malignancies. World J Gastrointest Oncol 2010;2:31-35.

14 Verwaal VJ, Van Tinteren H, Ruth SV, Zoetmulder AN: Toxicity of cytoreductive surgery and hyperthermic intra-peritoneal chemotherapy. J Surg Oncol 2004;85:61-67.

15 Kerscher AG, Mallalieu J, Pitroff A, Kerscher F, Esquivel J: Morbidity and mortality of 109 consecutive cytoreductive procedures with hyperthermic intraperitoneal chemotherapy (HIPEC) performed at a community hospital. World J Surg 2010;34:62-69. 
Bielen et al.: Rhabdomyolysis after Cytoreductive Surgery and Hyperthermic Intraperitoneal Chemotherapy: A Case Report

Glockzin G, Schlitt HJ, Piso P: Peritoneal carcinomatosis: patient selection, perioperative complications and quality of life related to cytoreductive surgery and hyperthermic intraperitoneal chemotherapy. W J Surg Oncol 2009;7:5.

-17 Glassman DT, Merriam WG, Trabulsi EJ, Byrne D, Gomella L: Rhabdomyolysis after laparoscopic nephrectomy. JSLS 2007;11:432-437.

18 Dornas de Oliveira L, Diniz MT, Diniz MD, Savassi-Rocha AL, Camargos ST, Cardoso F: Rhabdomyolysis after bariatric surgery by Roux-en-Y gastric bypass: a prospective study. Obes Surg 2009;19:1102-1107.

19 Alterman I, Sidi A, Azamfirei L, Copotoiu S, Ezri T: Rhabdomyolysis: another complication after prolonged surgery. J Clin Anesth 2007;19:64-66.

20 Ha TK, Choi YY, Song SY, Kwon SJ: F18-fluorodeoxyglucose-positron emission tomography and computed tomography is not accurate in preoperative staging of gastric cancer. J Korean Surg Soc 2011;81:104-110.

21 Portilla AG, Shigeki K, Dario B, Marcello D: The intraoperative staging systems in the management of peritoneal surface malignancy. J Surg Oncol 2008;98:228-231.

22 Elias D, Souadka A, Fayard F, Mauguen A, Dumont F, Honore C, Goere D: Variation in the peritoneal cancer index scores between surgeons and according to when they are determined (before or after cytoreductive surgery). Eur J Surg Oncol 2012;1-6.

-23 Yang XJ, Li Y, Yonemura Y: Cytoreductive surgery plus hyperthermic intraperitoneal chemotherapy to treat gastric cancer with ascites and/or peritoneal carcinomatosis: Results from a Chinese center. J Surg Oncol 2010;101:457-464.

24 Gill RS, Al-Adra D, Nagendran J, Campbell S, Shi X, Haase E, Schiller D: Treatment of gastric cancer with peritoneal carcinomatosis by cytoreductive surgery and HIPEC: a systematic review of survival, mortality and morbidity. J Surg Oncol 2011;104:692-698.

25 National Cancer Institute: Common Terminology Criteria for Adverse Events v4.0. NCI, NIH, DHHS. May 29, 2009 NIH publication \# 09-7473.

26 Casado-Adam A, Alderman R, Stuart A, Chang D, Sugarbaker PH: Gastrointestinal complications in 147 consecutive patients with peritoneal surface malignancy treated by cytoreductive surgery and perioperative intraperitoneal chemotherapy. J Surg Oncol 2011;2011:468698.

-27 Smeenk RM, Verwaal VJ, Zoetmulder FA: Learning curve of combined modality treatment in peritoneal surface disease. Br J Surg 2007;94:1408-1414.

28 Piso P, Glockzin G, Von Breitenbuch P, Popp FC, Dahlke MH, Schlitt HJ, Nissan A: Quality of life after cytoreductive surgery and hyperthermic intraperitoneal chemotherapy for peritoneal surface malignancies. J Surg Oncol 2009;100:317-320.

-29 McQuellon RP, Russel GB, Shen P, Stewart JH, Saunders W, Levine EA: Survival and health outcomes after cytoreductive surgery with intraperitoneal hyperthermic chemotherapy for disseminated peritoneal cancer of appendiceal origin. Ann Surg Oncol 2008; 15:125-133.

- 30 Guis S, Mattei JP, Cozzone PJ, Bendahan D: Pathophysiology and clinical presentations of rhabdomyolysis. Joint Bone Spine 2005;72:382-391. Allison RC, Bedsole DL: The other medical causes of rhabdomyolisis. Am J Med Sci 2003;326:79-88. Wrogemann K, Pena SD: Mitochondrial calcium overload: a general mechanism for cell-necrosis in muscle diseases. Lancet 1976;1:672-674.

33 Bosch X, Poch E, Grau JM: Rhabdomyolisis and acute kidney injury. N Engl J Med 2009;361:62-72.

-34 Chung JH, Ahn KR, Park JH, et al: Lower leg compartment syndrome following prolonged orthopedic surgery in the lithotomy position. Korean J Anesthesiol 2010;59:S49-S52.

Khan FY: Rhabdomyolysis: a review of the literature. Neth J Med 2009;67:272-283. Mubarak SJ, Pedowitz RA, Hargens AR: Compartment syndromes. Curr Orthop 1989;3:36-40. Zappa J, Sugarbaker PH: Compartment syndrome of the leg associated with lithotomy position for cytoreductive surgery. J Surg Oncol 2007;96:619-623.

38 Filis D, Daskalakis M, Askoxylakis I, Metaxari M, Melissas J: Rhabdomyolysis following laparoscopic gastric bypass. Obes Surg 2005;15:1496-1500.

-39 Dakwar E, Rifkin SI, Volcan IJ, Goodrich JA, Uribe JS: Rhabdomyolysis and acute renal failure following minimally invasive spine surgery. J Neurosurg Spine 2011;14:785-788.

-40 Faintuch J, de Cleva R, Pajecki D, Garrido AB, Cecconello I: Rhabdomyolysis after gastric bypass: severity and outcome patterns. Obes Surg 2006;16:1209-1213.

-41 Torres-Villalobos G, Kimura E, Mosqueda JL, Garcia-Garcia E, Dominguez-Cherit G, Herrera MF: Pressureinduced rhabdomyolysis after bariatric surgery. Obes Surg 2003;13:297-301.

-42 Lagandré S, Arnalsteen L, Vallet B, et al: Predictive factors for rhabdomyolysis after bariatric surgery. Obes Surg 2006;16:1365-1370.

43 Harris K, Walker PM, Mickle DA, et al: Metabolic response of skeletal muscle to ischemia. Am J Physiol 1986;250(2 Pt 2):213-220.

44 Blaisdell FW: The pathophysiology of skeletal muscle ischemia and the reperfusion syndrome: a review. Cardiovasc Surg 2002;10:620-630.

45 De Freitas Carvalho DA, Valezi AC, Macedo de Brito E, Lacerdo de Souza JC, Masson AC, Matsuo T: Rhabdomyolysis after bariatric surgery. Obes Surg 2006;16:740-744. 


\section{Case Reports in Oncology}

\begin{tabular}{l|l}
\hline Case Rep Oncol 2013;6:36-44 & \\
\hline DOI: $\underline{10.1159 / 000346471}$ & $\begin{array}{l}\text { ○ 2013 S. Karger AG, Basel } \\
\text { www.karger.com/cro }\end{array}$ \\
\hline
\end{tabular}

Bielen et al.: Rhabdomyolysis after Cytoreductive Surgery and Hyperthermic Intraperitoneal Chemotherapy: A Case Report

46 Reisiger KE, Landman J, Kibel A, Clayman RV: Laparoscopic renal surgery and the risk of rhabdomyolysis: diagnosis and treatment. J Urol 2005;66(suppl 5A):29-35.

-47 Ettinger J, Marcilio de Souza CA, dos Santos-Filho PV, Azaro E, Mello CA, Fahel E, Batista PB:

Rhabdomyolysis: diagnosis and treatment in bariatric surgery. Obes Surg 2007;17:525-532.

-48 Vanholder R, Sever MS, Erek E, Lameire N: Rhabdomyolysis. J Am Soc Neph 2000;11:1553-1561.

-49 Zager RA: Studies of mechanisms and protective maneuvers in myoglobinuric acute renal injury. Lab Invest 1989;60:619-629.

50 Better OS, Stein JH: Early management of shock and prophylaxis of acute renal failure in traumatic rhabdomyolysis. N Eng J Med 1990;322:825-829.

51 Brown CV, Rhee P, Chan L, Evans K, Demetriades D, Velmahos GC: Preventing renal failure in patients with rhabdomyolysis: do bicarbonate and mannitol make a difference? J Trauma 2004;56:1191-1196.

-52 Ettinger J, dos Santos-Filho PV, Azaro E, Melo CA, Fahel E, Batista PB: Prevention of rhabdomyolysis in bariatric surgery. Obes Surg 2005;15:874-879.

53 Pfeffer SD, Halliwill JR, Warner MA: Effects of the lithotomy position and external compression on the lower leg muscle compartment pressure. Anes 2001;95:632-636.

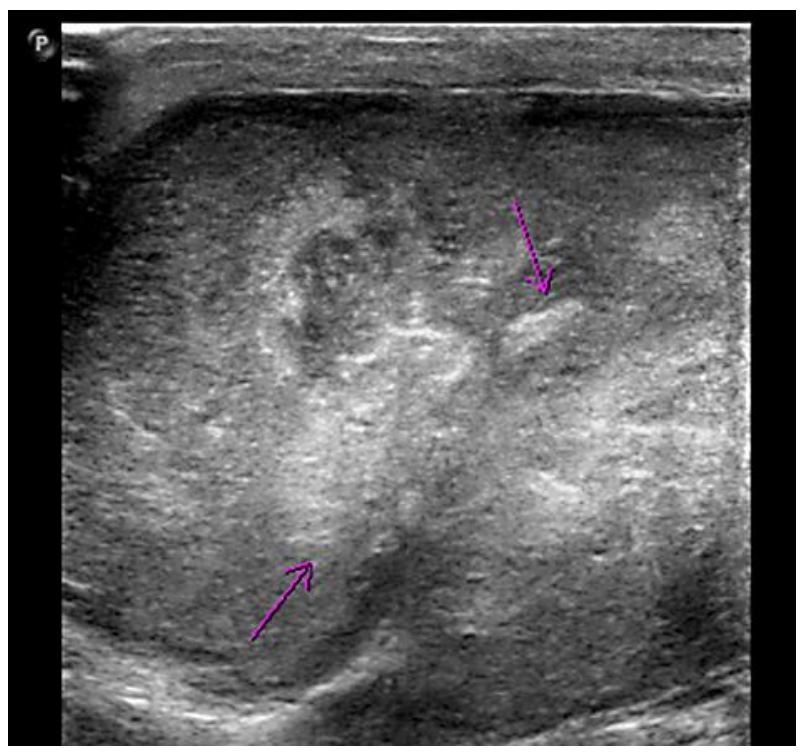

Fig. 1. Ultrasound image of the proximal gastrocnemius muscles in the axial plane: collection with hyperand hypoechoic areas (arrows), suggesting abscess formation. 


\section{Case Reports in Oncology}

Case Rep Oncol 2013;6:36-44

DOI: $\underline{10.1159 / 000346471}$

Bielen et al: Rhabdomyolysis after Cytoreductive Surgery and Hyperthermic

Intraperitoneal Chemotherapy: A Case Report

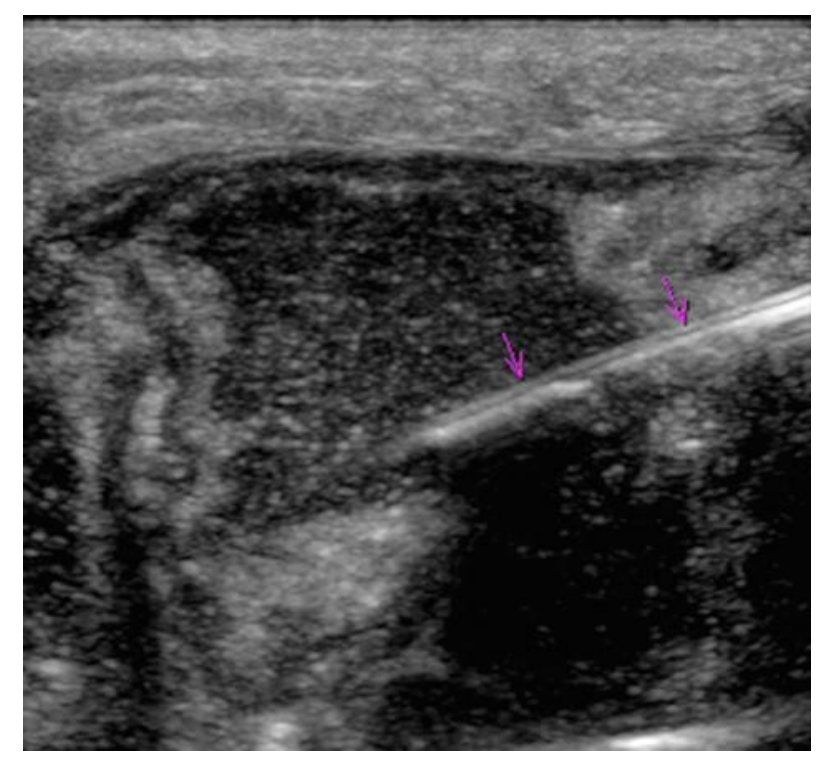

Fig. 2. Ultrasound image of an 18-gauge $\mathrm{Chiba}^{\circledR}$ puncture needle within the proximal gastrocnemius muscles in the axial plane. Ultrasonography-guided puncture of the abscess was followed by insertion of an $8 \mathrm{~F}$-pigtail catheter to drain the collection. This procedure was performed in both legs.

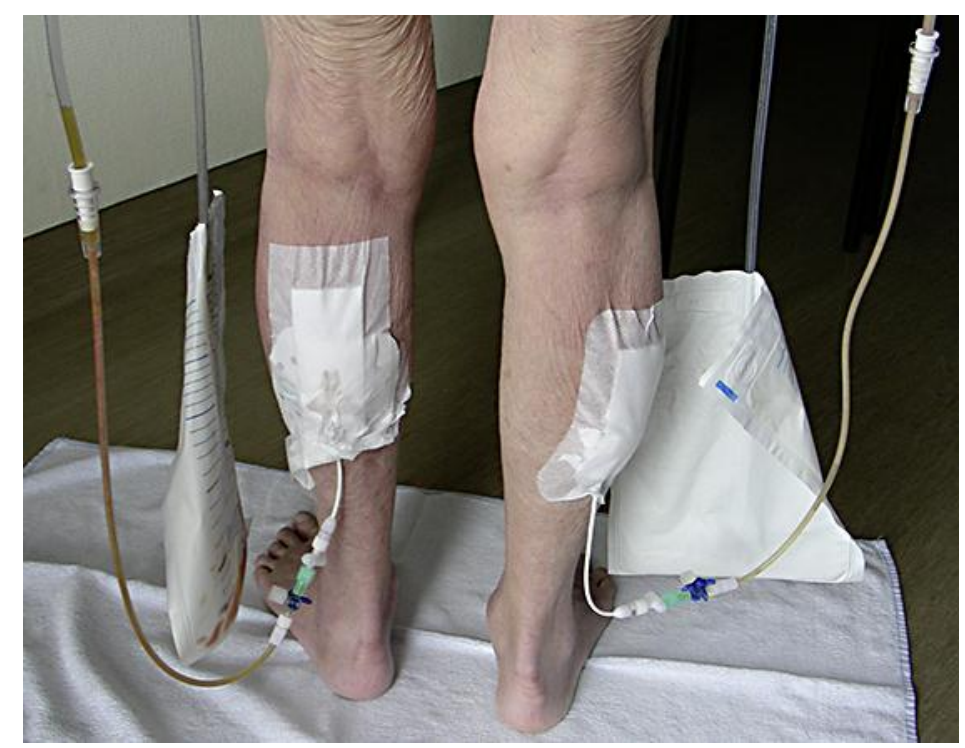

Fig. 3. 8F-pigtail catheters draining the sterile abscesses in the proximal gastrocnemius muscles. 